\title{
Research Paper \\ The Relationship Between Self-Efficacy and Quality of Life Among Elderly People
}

\author{
Javad Shaabani ${ }^{1}$, *Abolfazl Rahgoi ${ }^{2}$, Kian Nourozi ${ }^{1}$, Mahdi Rahgozar ${ }^{3}$, Mahdi Shaabani ${ }^{4}$ \\ 1. Department of Nursing, University of Social Welfare and Rehabilitation Sciences, Tehran, Iran. \\ 2. Research Centre on Aging, Department of Aging, University of Social Welfare and Rehabilitation Sciences, Tehran, Iran. \\ 3. Department of Biostatistics, University of Social Welfare and Rehabilitation Sciences, Tehran, Iran. \\ 4. Department of Nursing, Faculty of Nursing \& Midwifery, Hamadan University of Medical Sciences, Hamadan, Iran.
}

\begin{tabular}{|c|c|}
\hline $\begin{array}{l}\text { Use your device to scan } \\
\text { and read the article online }\end{array}$ & \\
\hline 口舟保回 & dtation: Shaabani J, Rahgoi A, Nourozi K, Rahgozar M, Shaabani M. [The Relationship Between Self-Efficacy and Quality of Life \\
\hline 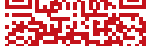 & Among Elderly People (Persian)]. Iranian Journal of Ageing. 2017; 11 (4):518-527. http://dx.doi.org/10.21859/sija-1104518 \\
\hline 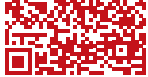 & dol: $:$ http://dx.doi.org/10.21859/sija-1104518 \\
\hline
\end{tabular}

Received: 22 Jun. 2016 Accepted: 08 Nov. 2016

Keywords:

Self-efficacy, Quality of life, Nursing home

\section{ABSTRACT}

Objectives Self-Efficacy is an important variable in the field of elderly, to which little attention has been paid. Quality of Life (QoL) is also one of the most important factors of human development. This study aimed to determine and investigate the relationship between self-efficacy and QoL among elders residing in Tehran nursing homes in 2015.

Methods \& Materials In this cross-sectional descriptive-analytic study, the statistical population comprised the residents of nursing homes, especially catering to the elderly, in Tehran. The sample size was 210 individuals, who were selected based on the research criteria. For the elderly people residing in elderly care centers, quota random sampling was performed, and random sampling in each nursing home was made proportionate to the number of elderly residents therein. General self-efficacy scale (GSE10), elderly quality of life questionnaire (LIPAD), and demographic questionnaire were utilized to collect data. The tools were investigated in terms of reliability. The elderly residents' average (SD) age was 78.66 (11.78) years. After sampling and gathering the questionnaires, the collected data were entered into SPSS Version18, and analyzed with descriptive indexes and analytic tests, including frequency, mean, standard deviation, Chi-square test, Kolmogorov-Smirnov test, ANOVA, and independent t test.

Results The findings showed that the mean (SD) score of self-efficacy among the elder residents of Tehran nursing homes was 17.68 (4.00), and the mean (SD) score of QoL was 27.88 (9.80). There is no significant correlation between self-efficacy and demographic characteristics such as age, sex, or marital status, but there was a significant correlation between the education level $(P=0.042)$ and self-efficacy. There also happened to be a significant correlation between $\mathrm{QoL}$, and age $(P=0.047)$ and education level $(P=0.038)$. Again, there was no significant correlation between QoL, and sex and marital status. In this particular research, the confidence level was considered as $95 \%$.

Conclusion According to the results of the study, self-efficacy of residential elderlies and their quality of life was low. Increase in self-efficacy among the elderly residents of the nursing homes led to a rise in their QoL.

\section{* Corresponding Author:}

Abolfazl Rahgoi, MSc.

Address: University of Social Welfare and Rehabilitation Sciences, Kodakyar Ave., Daneshjoo Blvd., Evin, Tehran, Iran.

Tel: +98 (021) 22180036

E-mail: rahgouin@yahoo.com 


\title{
ارتباط خودكار آمدى و كيفيت زندتى سالمندان مقيم آسايشكاههاى شهر تهران
}

\author{
جواد شعبانى'، "ابوالفضل رهكوى"، كيان نوروزى'، مهلى رهكذر"، مهدى شعبانى"
}

ا- كروه يرستارى، دانشعاه علوم بهزيستى و توانبخشى، تهران، يران.

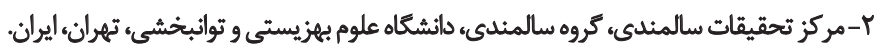

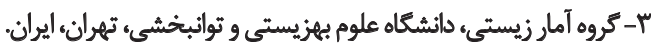

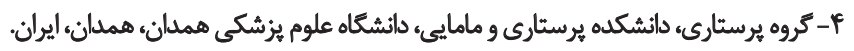

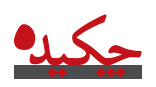

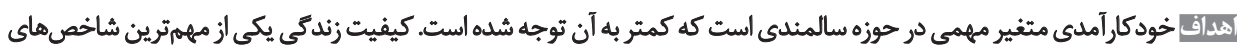

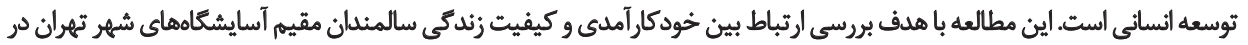

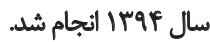

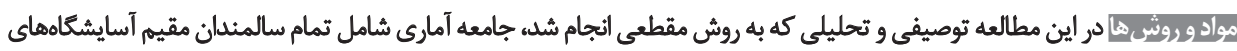

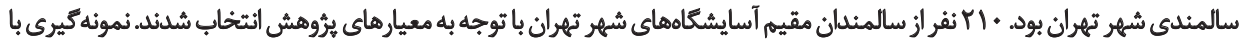

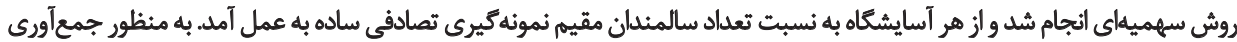

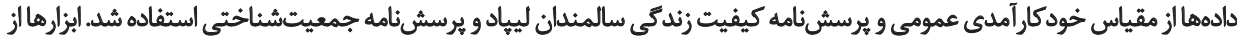

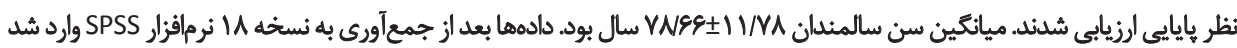

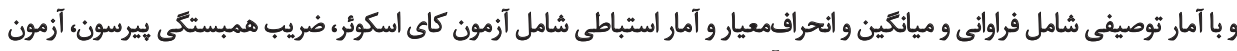

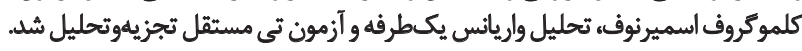

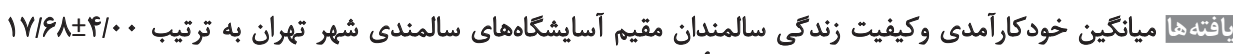

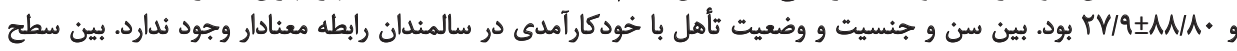

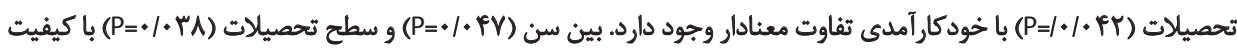

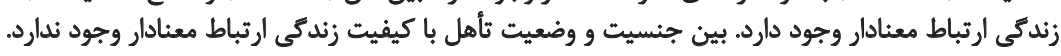

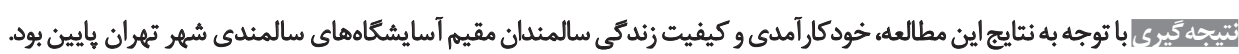

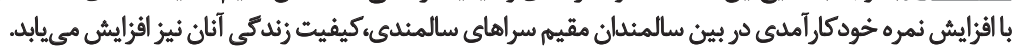

تاريخ دريافت: · تير هوسا

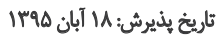

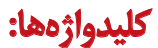
خودكارآمدى، كيفيت زندكى، خانه سالمندان

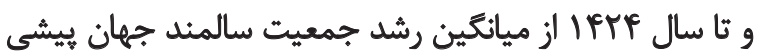

مقدمه

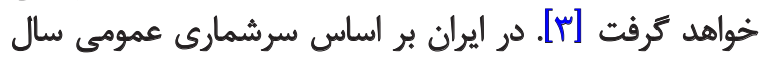
كNA

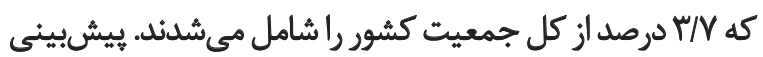

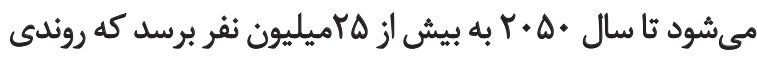

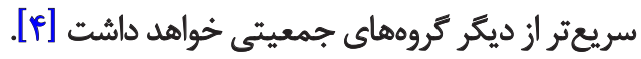

امروزه تعداد سالمندان به سرعت در حال افزايش است. توجه

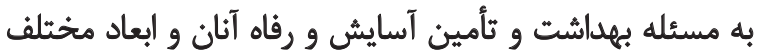

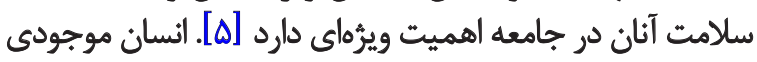

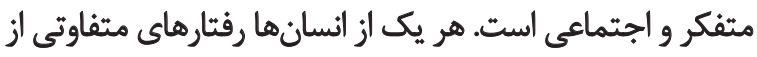

سالمندى فرايندى همكاني است كه از لحظه لقاح آغاز مي شود

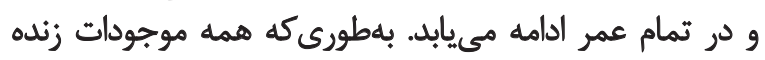

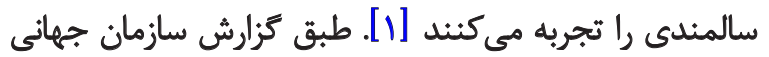

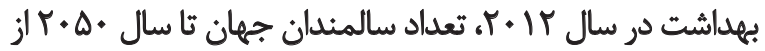

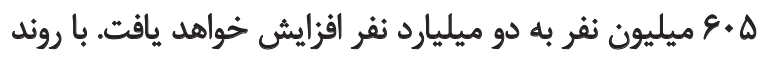

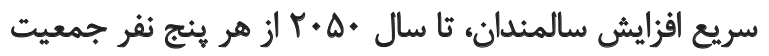

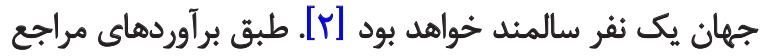

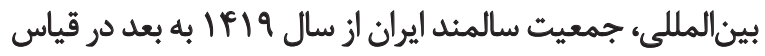

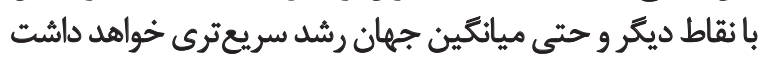




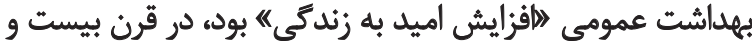

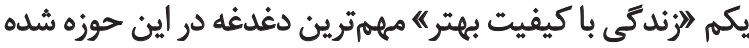

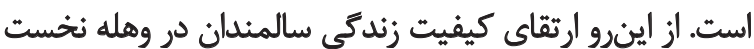

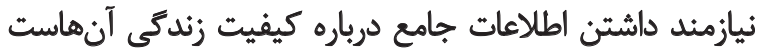

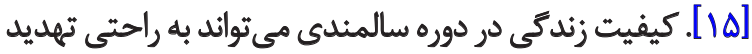

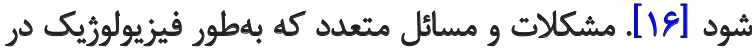

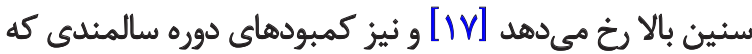

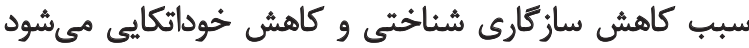

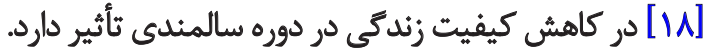

خودكارآمدى و كيفيت زندگى در دوران بيرى نسبت به به بازي

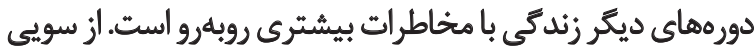

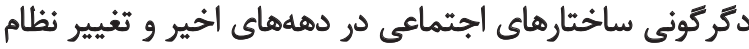

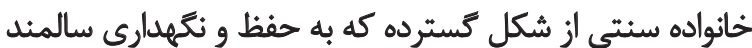

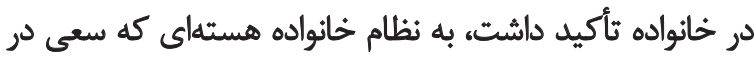

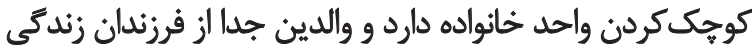

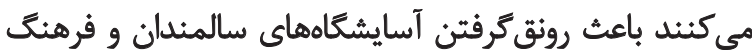

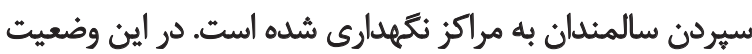

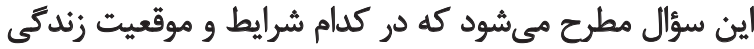

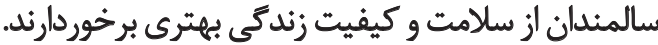

تحقيقات نشان مي دهد محيط زندكى سالمندان عاملى بسيار

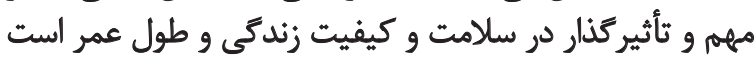

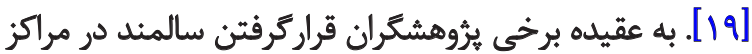

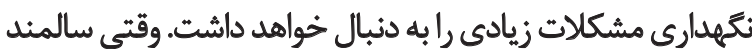

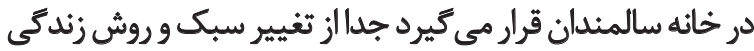

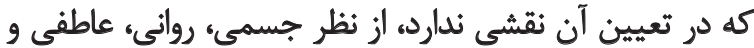

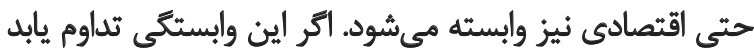

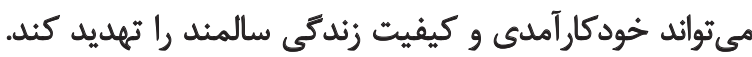

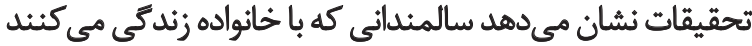

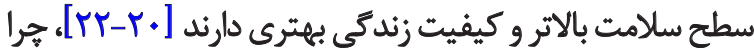

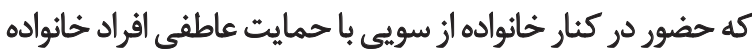

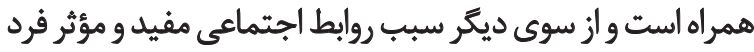

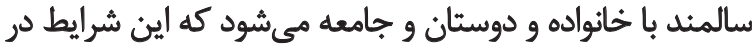

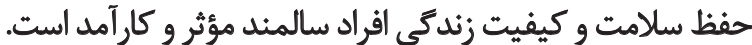

يافتههاى محدودى به ثأثير مثبت نكّهدارى افراد سالخورده

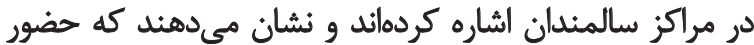

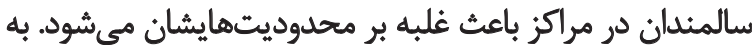

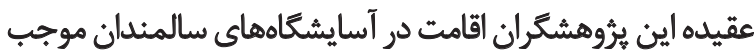

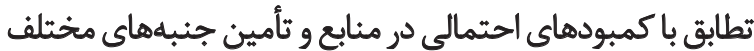

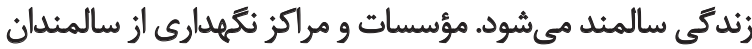

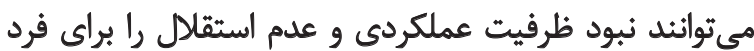

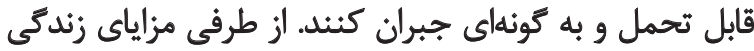

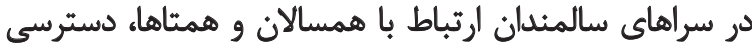

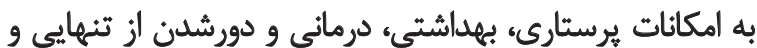

خود بروز مى دهند [ع]. عوامـل متعددى در شكل كيرى رفتئار

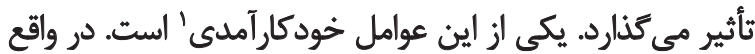

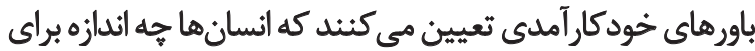

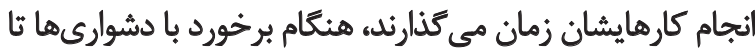

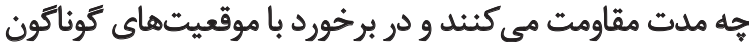

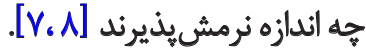

خودكارآمدى درجهاي از احساس تسلط فرد درباره توانايى

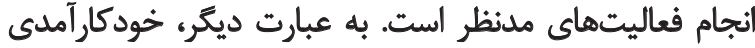

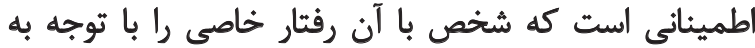

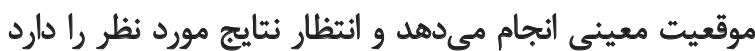

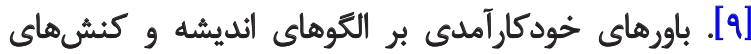

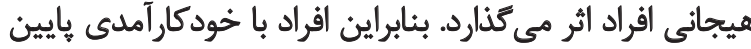

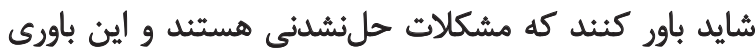

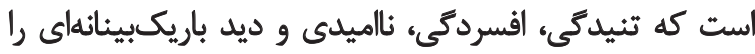

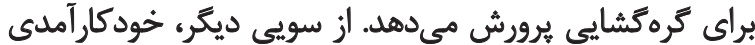

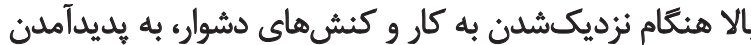

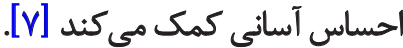

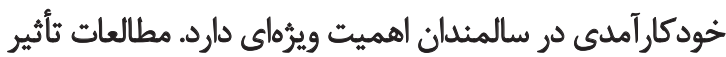

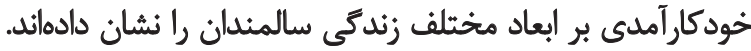

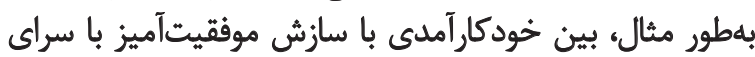

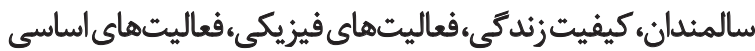

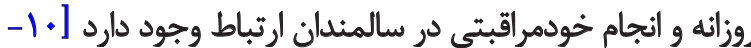

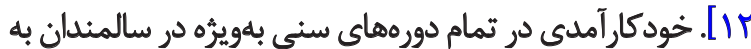

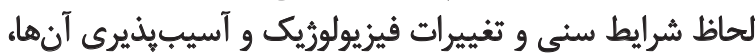
بحث وسيعى را بين متخصصان ايجاد كرده است.

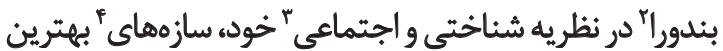

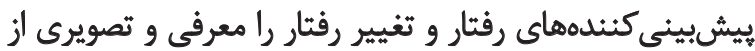

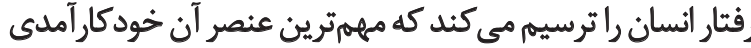

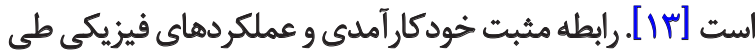

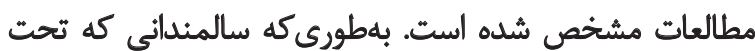

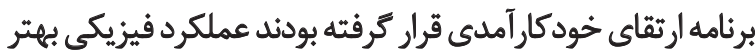

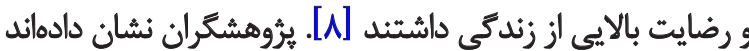

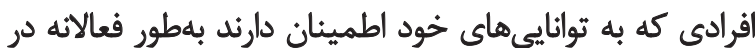

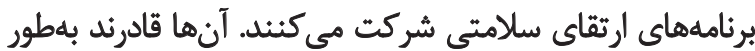

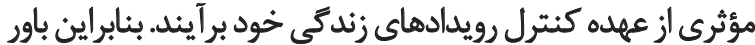

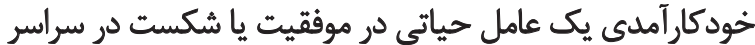

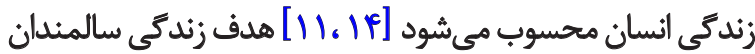

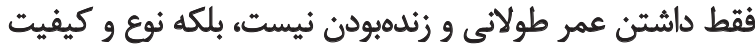

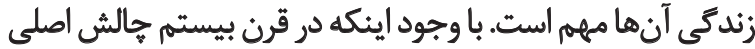

1. Self Efficacy

2. Bandura

3. Social Cognitive Theory

4. Constructs 
يك از نمونهها در خواندن و يا درك سؤالهاي يرسشنامهنها

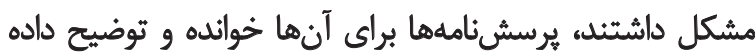

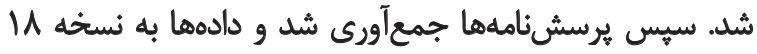

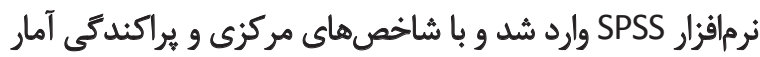

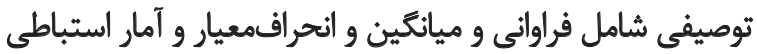

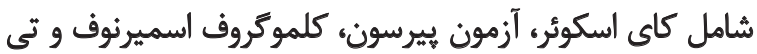

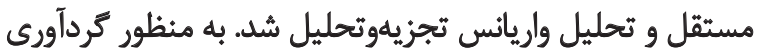

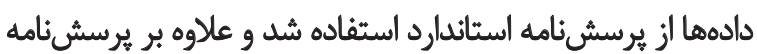

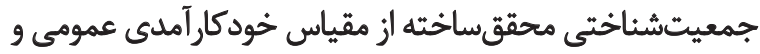
يرسشنامه كيفيت زندكى سالمندان ليهاد استفاده شد.

\section{مقياس خودكار أمدى عمومى (+ (GSE-1)}

اين مقياس را شوآرزره و اورشليم" ساختند كه در ايران در

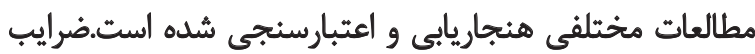

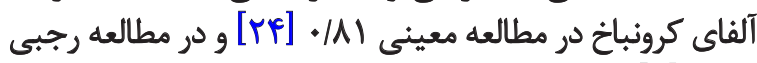

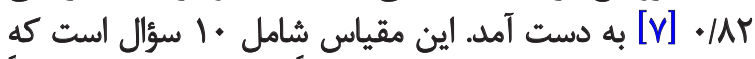

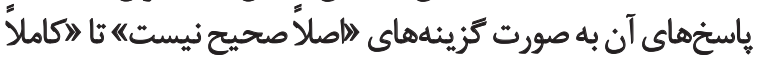

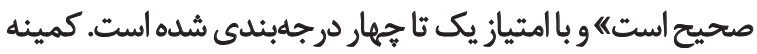

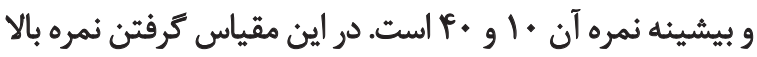

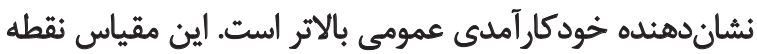

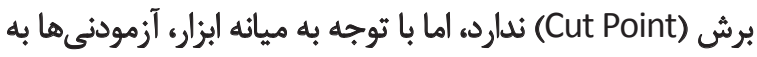

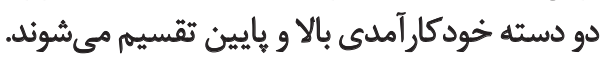

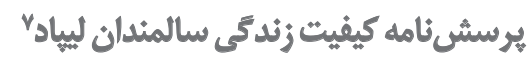

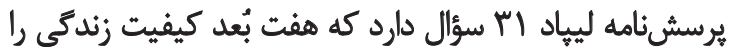

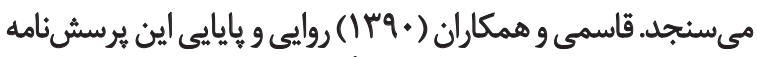

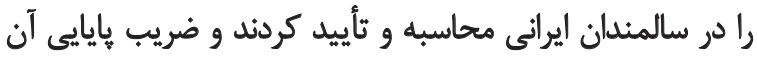

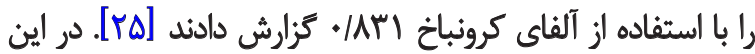

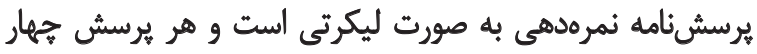

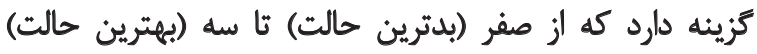

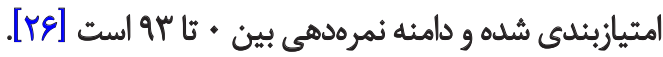

يافتهاهنا

در اين مطالعه · · M سالمند شركت كردند. فراوانى زنان

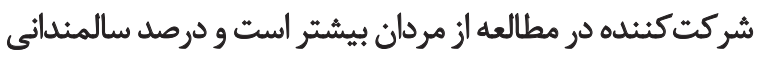

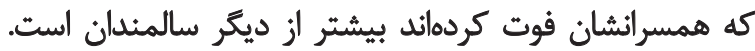

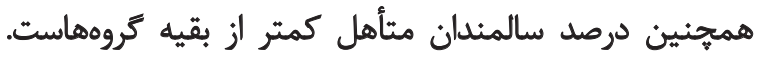

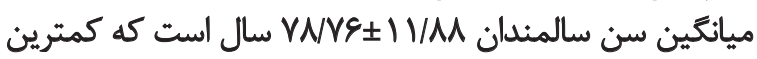

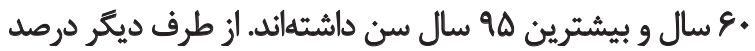
سالمندانى كه بي سوادند (بال سالمند) بيشتر از ديكر كروهاست

5. Schwarzer

6. Jerusalem

7. Leipad
افسردكى است كه مي تواند در حفظ سلامت و كيفيت زندكى و

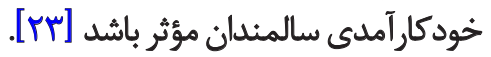

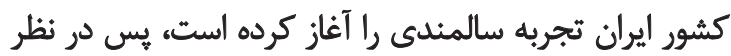

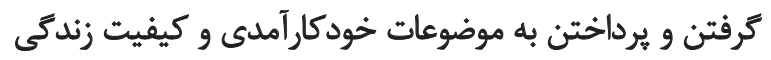

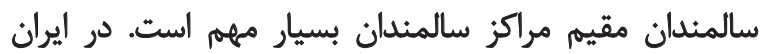

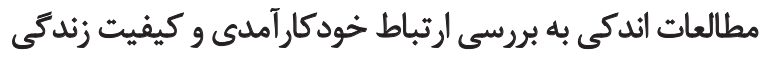

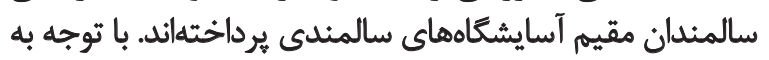

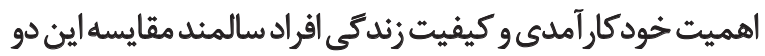

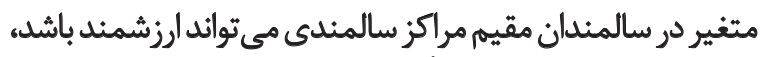

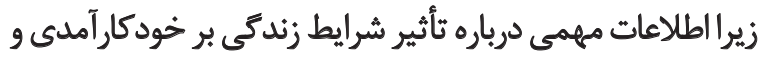
كيفيت زندگى سالمندان ارائه مى دهد.

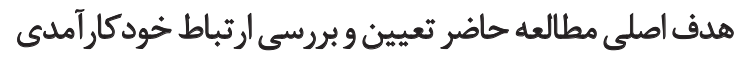

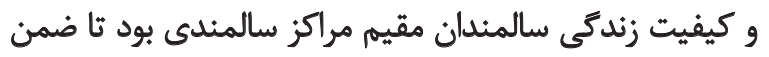

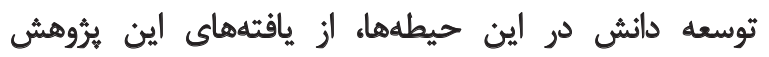

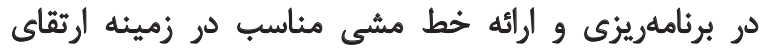

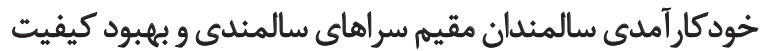
زندكى آنان استفاده شود.

\section{روش مطالعه}

اين مطالعه از نوع توصيفى تحليلى بود كه به روش مقطعى تمام مقام

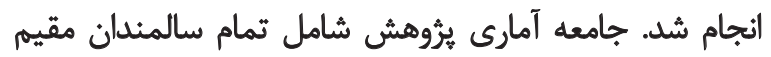

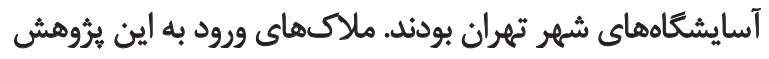

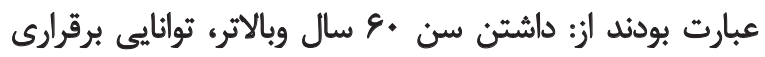

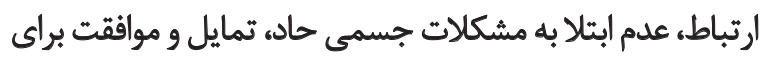

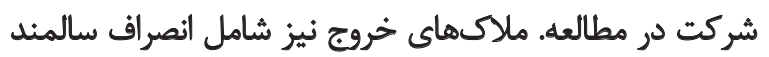

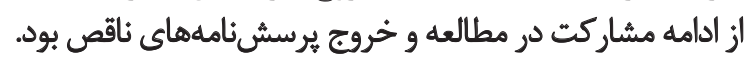

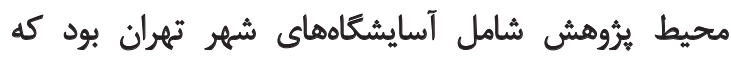

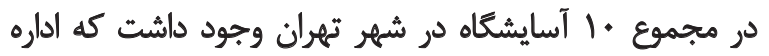

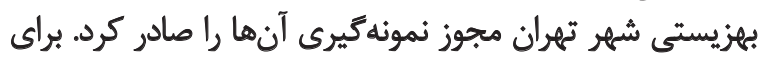

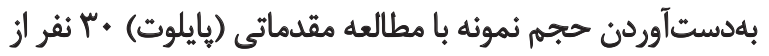

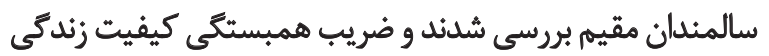

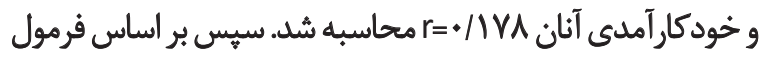

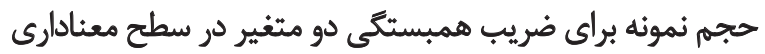

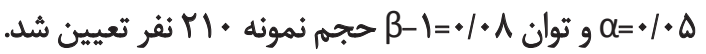

هجوز نمونه كيرى از كميثه اخلاق دو بثوهش دانشّكاه علوم بهزيستى و توانبخشى تهران و سازمان بهزيستى شهر تهران

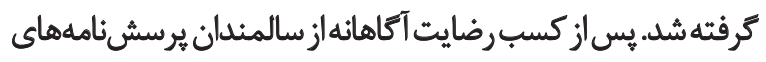

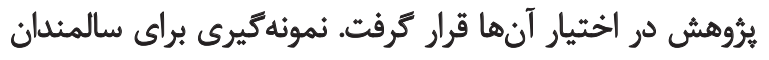

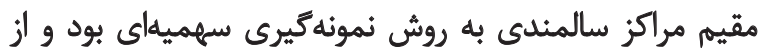

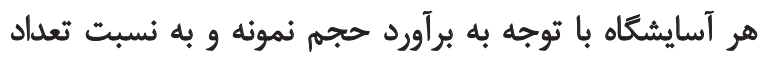

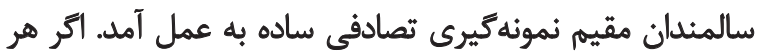


مقدار Pربوط به آزمون كلموكروف اسميرنوف در نمرههاى

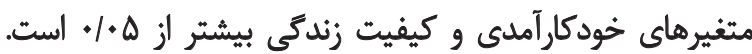

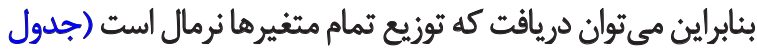

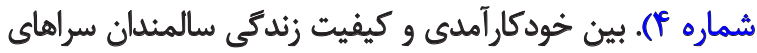

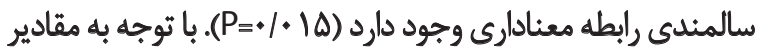

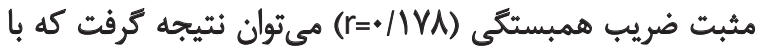

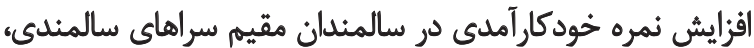

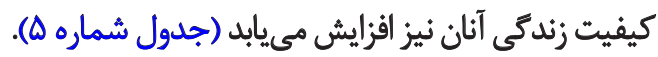

با توجه به ضريب همبستگى بين سن با خودكارآمدى

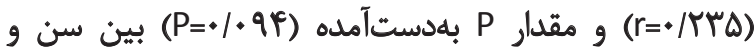

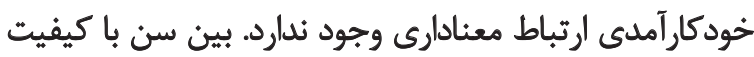

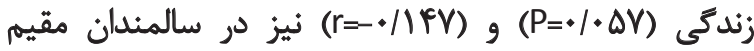

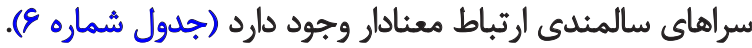

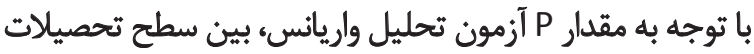

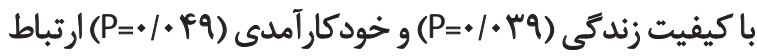

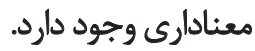

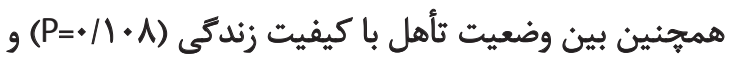

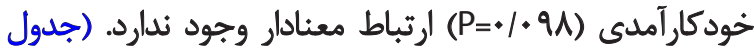

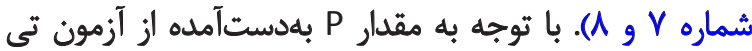

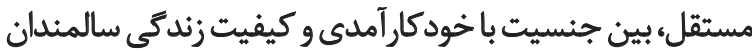

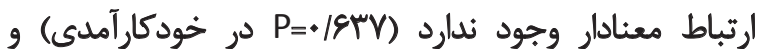

و فقط 10 سالمند تحصيلات دانشكاهى دارند. بيشتر سالمندان

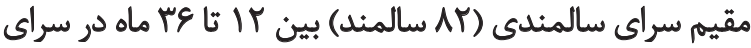

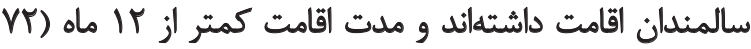
سالمند) در رتبه بعدى قرار دارد (جدول شماره ل).

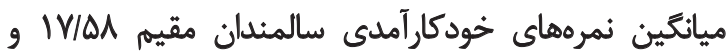

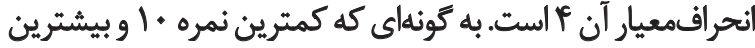

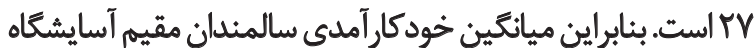

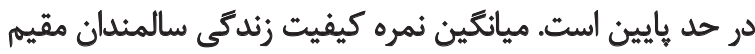

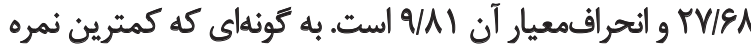

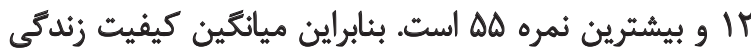

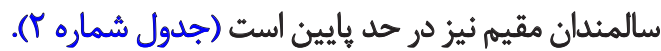
در ادامه توزيع براكندكى خودكارآمدى و كيفيت زندگى به به

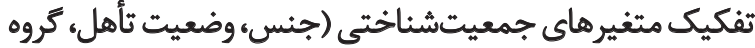

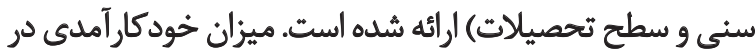

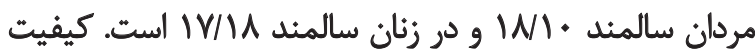

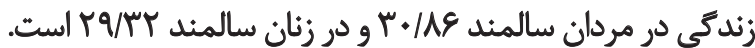

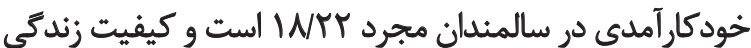

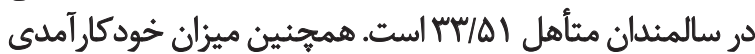

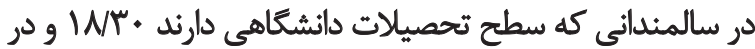

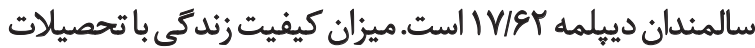

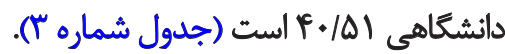

جدول ا. اطلاعات جمعيتشئاختى سالمندان.

\begin{tabular}{|c|c|c|c|}
\hline \multicolumn{2}{|c|}{ سالمندان مقيم } & \multirow{2}{*}{\multicolumn{2}{|c|}{ متغير }} \\
\hline يرصد & تعداد & & \\
\hline$s g / f$ & if. & زن & \\
\hline$m / \varepsilon$ & v. & مرد & جنسيت \\
\hline $1.0 \%$ & ri. & جمع & \\
\hline$r / r$ & PD & هجرد & \\
\hline$\Delta \Delta$ & 118 & همسر فوتششده & \\
\hline$I f / V$ & r. & هطلقه & وضعيت تأهل \\
\hline$q / r$ & 19 & متأهل & \\
\hline $1.0 \%$ & M. & جمع & \\
\hline$M \pi /$. & $\Delta$. & ع r. & \\
\hline$W_{F}$ & f. & 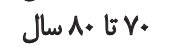 & \\
\hline eV/o & $1 \ldots$ & .1 تأ .9 سال & كروه سني \\
\hline $11 /$ & r. & بيشتر از +9 سال & \\
\hline $1++1+$ & r. & جمع & \\
\hline ff & १५ & بىسواد & \\
\hline res & ve & سيكل & \\
\hline ir & rV & دييلم & سطح تحصيلات \\
\hline $8 / 9$ & 10 & دانشكاهى & \\
\hline $1 . . \%$ & M. & جمع & \\
\hline
\end{tabular}


جدول r. مقدار كميئه، بيشيئه، ميانئين و أنحر افسمعيار خودكار آمدى و كيفيت زندكي سالمندان مقيه.

\begin{tabular}{|c|c|c|c|c|}
\hline انحرافمعيار & مياتكين & بيشينا & كمينه & هتغير \\
\hline$\%$ & $I V / \Delta A$ & rV & 1. & خودوكار أملىى \\
\hline $9 / 11$ & TVIFA & $\Delta \Delta$ & ir & كيفيت زندكى \\
\hline
\end{tabular}

$\stackrel{1}{2}$

جدول ب. توزيع يراكندكى در ارتباط با ميزان خودكارآمدى و كيفيت زندكى در سالمثلدان به تفكيك جنسيت، وضعيت تأهل، سطح تحصيلات و سن.

\begin{tabular}{|c|c|c|c|c|c|}
\hline انحرافمعيار كيفيت & ميانكين كيفيت زندكى & خودكارآمديى & ميانعين خودكارآمدى & شاخص & مثتغير \\
\hline IP/aP & TQMT & $r / q$. & $I V / M$ & ن & \multirow{2}{*}{ جنسيت } \\
\hline$|\Delta / 1|$ & $r \cdot M e$ & $\varphi / .1$ & WI. & مرد & \\
\hline $\mid Q / \Delta V$ & rNAY & $\varphi / M$ & $I V / N$. & j & \multirow{2}{*}{ سن } \\
\hline $1 \pi / 9$. & $r q / 4$ & $M / 8 A$ & IV/a. & مرد & \\
\hline$|r / M|$ & MIMT & $F / M$ & WNT & مجرد & \multirow{4}{*}{ وضعيت تأهل } \\
\hline$|r / T|$ & $M V / M^{\prime}$ & $m / 9 \Delta$ & $W \cdot q$ & همسر فوتشده & \\
\hline $1 \notin / \pi \Delta$ & TNAD & $r / m$. & $18 / 1$. & مطلقه & \\
\hline $10 / K E$ & $M T / \Delta)$ & $r / \varepsilon$. & IV/TO & متأهل & \\
\hline $10 / 9$. & rq/a. & $r / r$. & $10 / \Delta 9$ & بي سواد & \multirow{4}{*}{ سطح تحصيلات } \\
\hline$|\& / \pi|$ & $m+1$ & $r / q$ & $18 / 79$ & سيكل & \\
\hline $\mid r / r$ & rNV. & $r / r \mu$ & IV/AT & ديبلم & \\
\hline $\mathrm{V} / \mathrm{TA}$ & $r \cdot(\Delta)$ & $r / r$ & $\mathbb{N}^{\mathbf{H}}$. & دانشكاهى & \\
\hline
\end{tabular}

几

$$
\text { تحصيلات ديكَ بيشُتر بود. }
$$

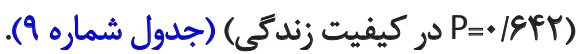

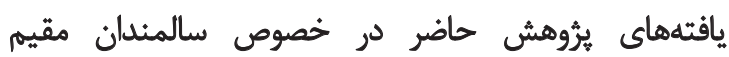

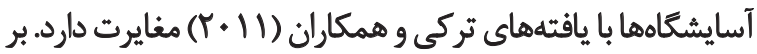

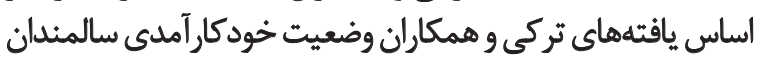

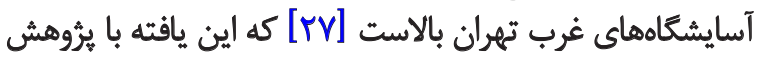

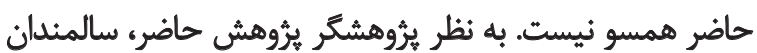

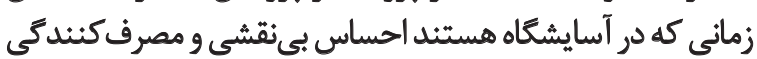

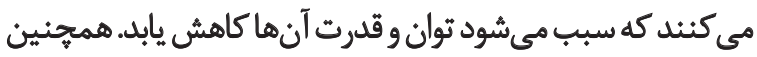

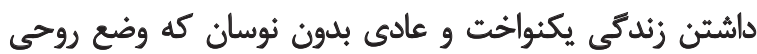

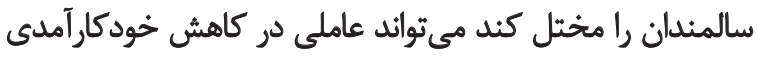

با توجه به يافتهماى بلدستآمده ميانتين نمرههاى

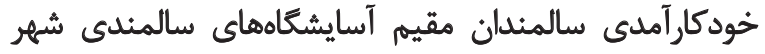

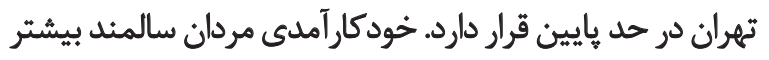

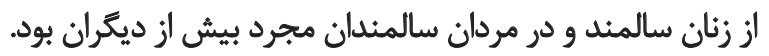

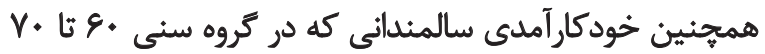

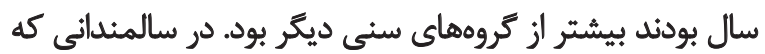

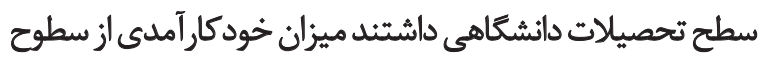

جدول F. آزمون تعيين نرمالبونن متغيرهاى خودكارآمدى و كيفيت زندكى سالمثدان مثيم آسايشكاههاى سالمثدى.

\begin{tabular}{|c|c|c|}
\hline مقدار P P P & آماره كلموكروف اسميرنوف & متغير \\
\hline.$/ 1 P Q$ & WIFo & خودكار|آمدى \\
\hline 1.91 & I/THT & كيفيث زندكى \\
\hline
\end{tabular}

乩 
جدول ه. نتايج آزمون همبستكى بين خودكارآمدى و كيفيت زندكى سالمندان.

\begin{tabular}{|c|c|c|}
\hline \multicolumn{2}{|c|}{ كيفيت زندكى } & \multirow[b]{2}{*}{ متغير } \\
\hline Pقدار P P & ضريب همبستكى & \\
\hline $.1 \cdot 10$ &.$/ 1 \mathrm{ra}$ & خودكارآمدى \\
\hline
\end{tabular}

닌

جدول وا نتايج آزمون همبسئكى بين سن با خودكار آمدى و كيفيت زندكى سالمئدان.

\begin{tabular}{|c|c|c|}
\hline \multicolumn{3}{|c|}{ سن } \\
\hline Pقدار P P & ضريب همبستكى & متغير \\
\hline$\%$. Af & - MTS & خودارارآملى \\
\hline $.1 \cdot \mathrm{PV}$ & $-* / 1 T V$ & كيفيت زندكى \\
\hline
\end{tabular}

L

جدول V. مقايسه ميانكين (تحليل واريانس) سطح تحصيلات در سالمئدان.

\begin{tabular}{|c|c|c|}
\hline مقدار P & foار fol & مثغير \\
\hline $.1+4 q$ & $r / 19$ & خودكارألمدى \\
\hline$+1+49$ & r/aAr & كيفيت زندكى \\
\hline
\end{tabular}

L

جدول ^. مقايسه ميانكّين (تحليل واريانس) وضعيت تأهل سالمثدان.

\begin{tabular}{|c|c|c|}
\hline مقدار P & أهماره & هتغير \\
\hline .1 .41 & $r / \Delta \Delta$ & خودكار آمدى \\
\hline$\cdot / 1 \cdot 1$ & P/qPV & كيفيت زندكى \\
\hline
\end{tabular}

in

جدول و. نتايج آزمون تى مستقل براى مقايسه ميانتين جنسيت با خودكار آمدى و كيفيت زندكّى سالمندان.

\begin{tabular}{|c|c|c|c|c|c|}
\hline مقدار p p & t t آماره & انحرافـمعيار & مياتكّين & كروه & متغير \\
\hline \multirow{2}{*}{ - Ietr } & \multirow{2}{*}{ - prie } & $r / q$. & & نj & \multirow{2}{*}{ خودكارآمدى } \\
\hline & & $4 / .1$ & $|N|$. & هرد & \\
\hline \multirow{2}{*}{ - Metr } & \multirow{2}{*}{. IfIr } & $1 f / 97$ & rQ/MT & ij & \multirow{2}{*}{ كيفيت زندكى } \\
\hline & & $|\Phi / 1|$ & $P \cdot / N E$ & هرد & \\
\hline
\end{tabular}

L

مطالعهاى ارتباط خودكارآمدى عمومى و كيفيت زندكى را در

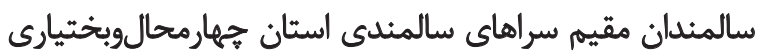

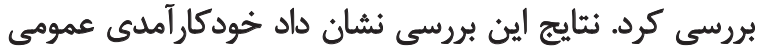

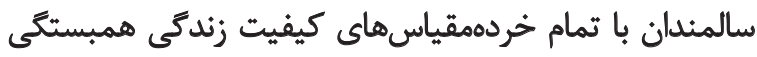

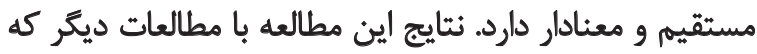

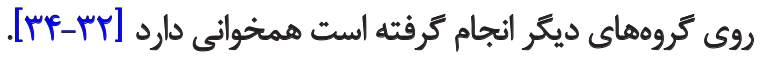

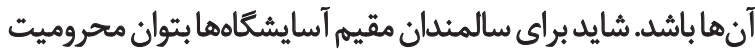
از خانواده را يكى از ديكر دلايل اين مسئله دانست.

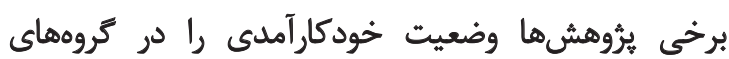

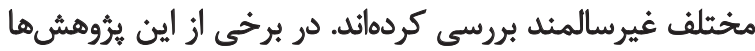

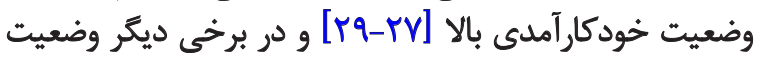

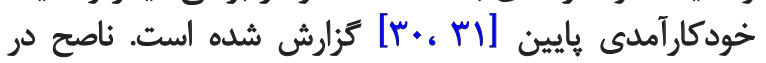


مرد بلهور معنادارى بهتر از كيفيت زندكى سالمندان زن بود.

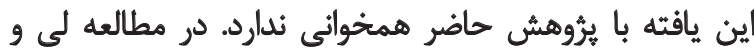

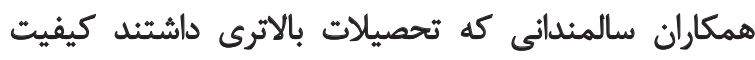

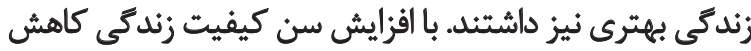

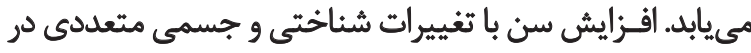

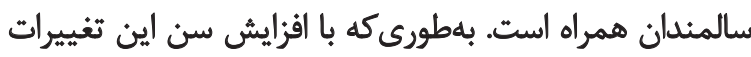

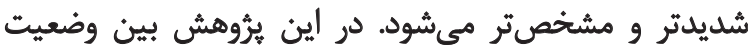

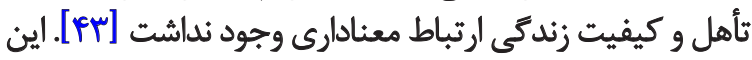

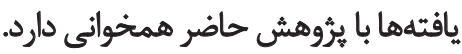

داشتن همسر در دوران سالمندى كام مؤثرى در تقويت روحيه

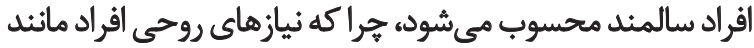

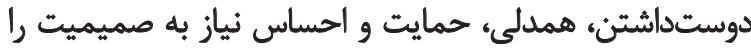

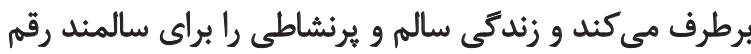

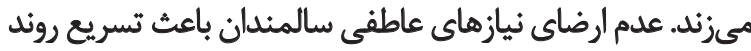

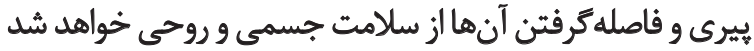

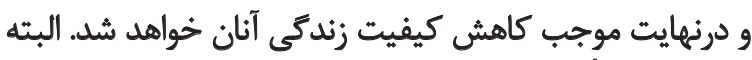

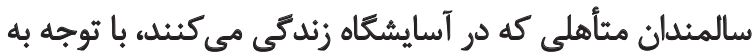

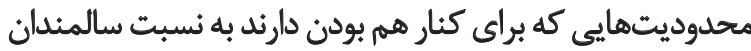

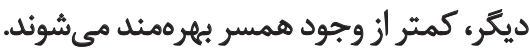

با توجه به يافتههاى اين يثروهش بين خودكار آمدى و كيفيت

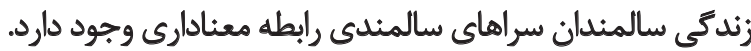

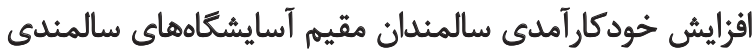

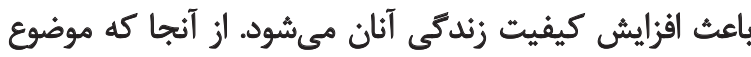

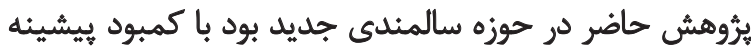

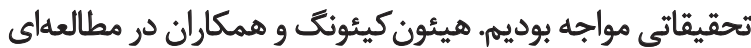

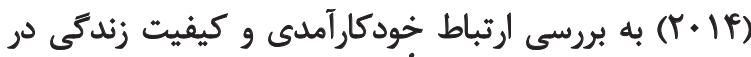
دادخ كار TFq

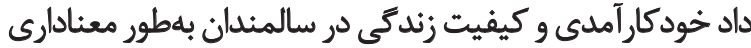

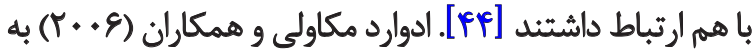

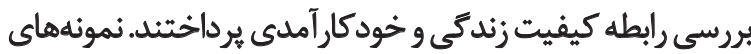

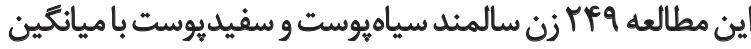

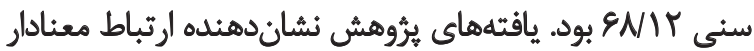

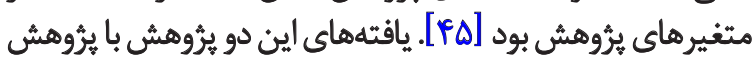
حاضر همنخوانى دارد.

\section{نتيجلكيرى نهايى}

زبر اساس يافتههاى برثوهش وضعيت خودكارآمدى و كيفيت

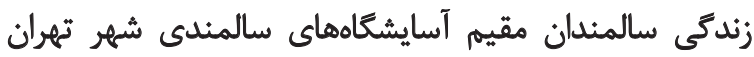

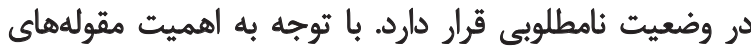

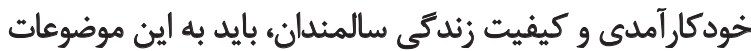

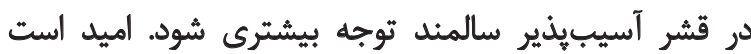

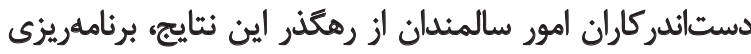

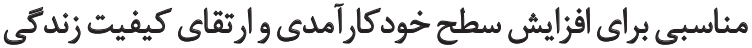

اسميت معتقد است خود كار آمدى بالا باعث افزايش اميد و انكيزه

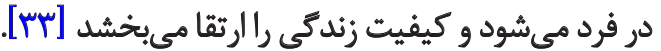

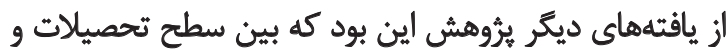

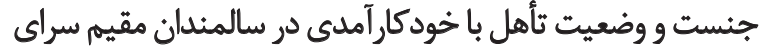

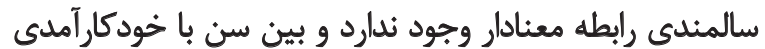

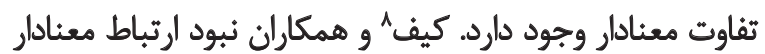

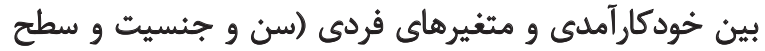

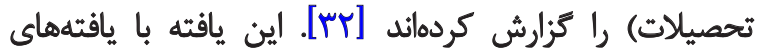

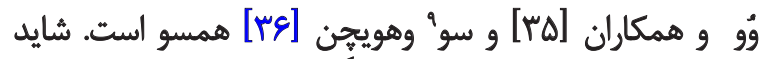

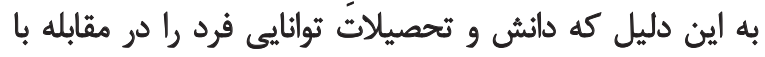

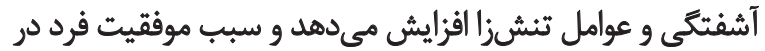

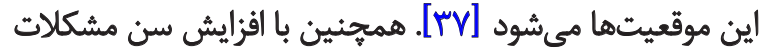

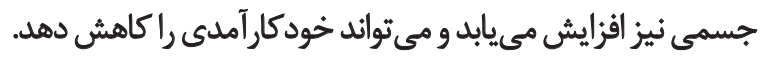

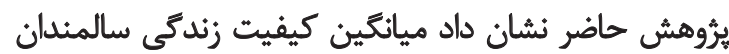

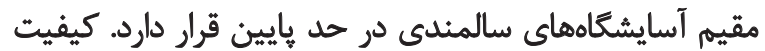

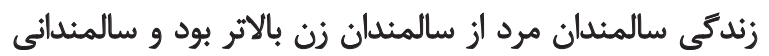

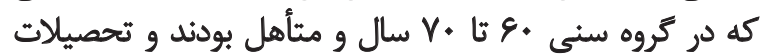

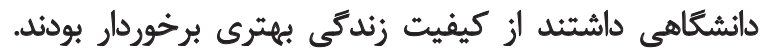

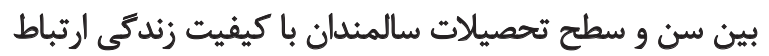

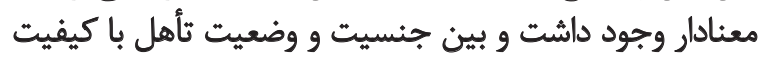

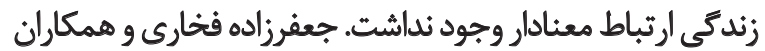

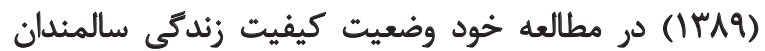

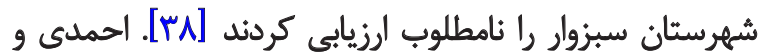

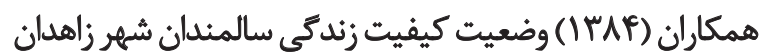

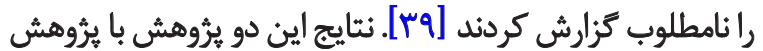

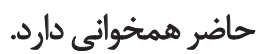

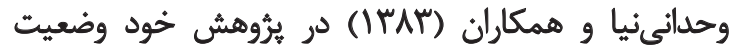

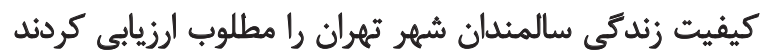

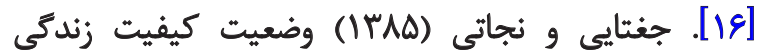

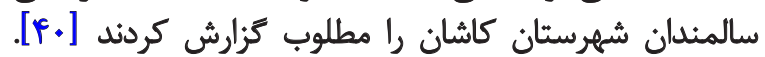

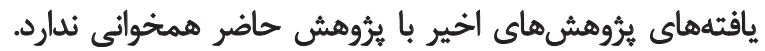

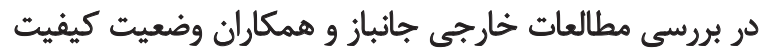

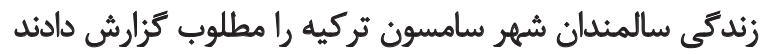

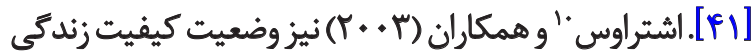

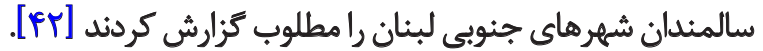

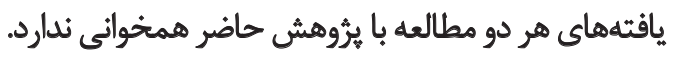
در مطالعه لى" و همكاران (9. (Y) بين جنسيت و كيفيت زندكى ارتباط معنادارى وجود داشت و كيفيت زندكى سالمندان 


\section{References}

[1] Abedi HA, Shahriari M, Alimohammadi N. [Nursing in elderly (Persian)]. Isfahan: Oroo; 2003

[2] World Health Organization. World health statistics. Geneva: World Health Organization; 2012.

[3] Mirzaei M, Shams-Ghahfarkhi M. [Demographic characteristics of the elderly population in Iran according to the census 1976-2006 (Persian)]. Iranian Journal of Ageing. 2007; 2(5):326-31

[4] Statistical Center of Iran. [Census of 2006 (Persian)]. Tehran: Statistical Center of Iran; 2006

[5] Habibi A, Nikpour S, Seyedoshohadaei M, Haghani H. [Health promoting behaviors and its related factors in elderly (Persian)] Iran Journal of Nursing. 2006; 19(47):35-48.

[6] Shariatmadari A. [Educational psychology (Persian)]. 11 ${ }^{\text {th }}$ ed. Tehran: Amir Kabir Publication; 2002.

[7] Rajabi G. [Reliability and validity of the General Self-Efficacy Beliefs Scale (GSE-10) comparing the psychology students of Shahid Chamrin University and Azad University of Marvdasht (Persian)]. New Thoughts on Education. 2006; 2(1):111-22.

[8] Rabiei L, Mostafavi F, Masoudi R, Hassanzadeh A. [Effects of family-centered interventions on empowerment of the elderly (Persian)]. Health System Research. 2012; 8(2):301-13.

[9] Akbaryboorang A, Aminyazdi SA. [Test-anxiety and self-efficacy: A study on the students of Islamic Azad University, Branch of Southern Khorasan (Persian)]. Ofogh-e-Danesh. 2009; 15(3):70-77.

[10] Bhupinder S, Rakhi U. Self-efficacy and well-being of adolescents. Journal of the Indian Academy of Applied Psychology. 2009; 35(2):227-32

[11] Chang S-H, Crogan NL, Wung S-F. The self-care self-efficacy enhancement program for Chinese nursing home elders. Geriatric Nursing. 2007; 28(1):31-6. doi: 10.1016/j.gerinurse.2006.11.006

[12] Hellstrom K, Lindmark B, Wahlberg B, Fugl-Meyer AR. Selfefficacy in relation to impairments and activities of daily living disability in elderly patients with stroke: a prospective investigation. Journal of Rehabilitation Medicine. 2003; 35(5):202-7.

[13] Zimmerman BJ. Self-efficacy: An essential motive to learn. Contemporary Educational Psychology. 2000; 25(1):82-91. doi: 10.1006/ceps.1999.1016

[14] Lev EL, Daley KM, Conner NE, Reith M, Fernandez C, Owen $\mathrm{SV}$. An intervention to increase quality of life and self-care self-efficacy and decrease symptoms in breast cancer patients. Scholarly inquiry for nursing practice. 2001; 15(3):277-94. PMID: 11871585

[15] Sajjadi H, Biglryian A. [Quality of life in elderly women in Kahrizak Hospice Charity (Persian)]. Payesh. 2006; 5(2):105-8,

[16] Vahdaninia M, Goshtasbi A, Montazeri A, Maftoun F. [Healthrelated quality of life in an elderly population in Iran: A population-based study (Persian)]. Payesh. 2005; 4(2):113-20.

[17] Habibi A, Nikpour S, Seyedoshohadaei M, Haghani H. [Quality of life and physical activity (Persian)]. Iran Journal of Nursing. 2007; 21(53):30-51.
سالمندان مقيم سراي سالمندى تدوين كنئد و ملاحظات لازم را أنجام دهند ثادر آينده شاهد زندئ سيى باكيفيت در سالمئدان باشيهم.

محدوديتها

به دليل محدودبودن نمونه يثروهش به سالمندان شهر تهران

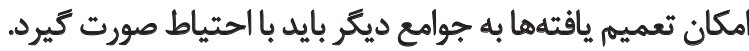

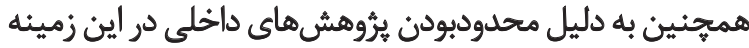

امكان مقايسه كامل يافتهها وجود نداشت. محددوديت ديت ديكر

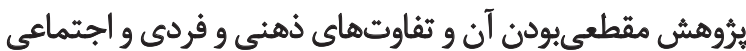

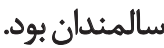

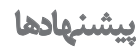

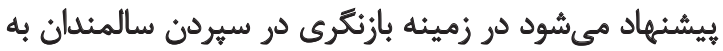

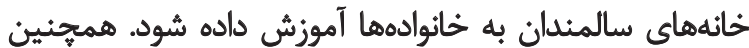

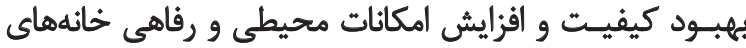

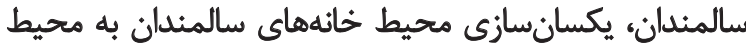

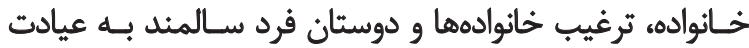

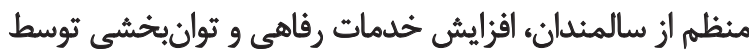

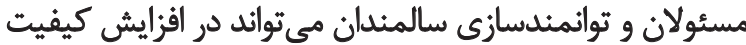
زندكى سالمندان سودمئد باشئ.

تششكر و قدرداني

اين مطالعه از طرح يُروهشى مصوب در مركز ثحقيقات

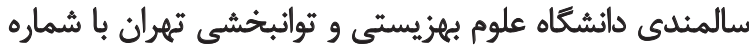

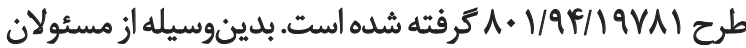

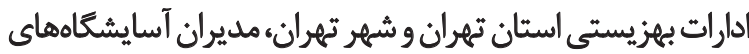

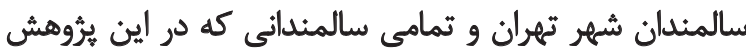

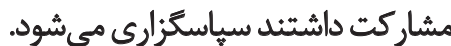


[18] Tajvar M, Farziyanpour F. [Elderly health and a review on different Aspects of their life (Persian)]. Tehran: Nasle Farda Publications; 2004.

[19] Winningham RG, Pike NL. A cognitive intervention to enhance institutionalized older adults' social support networks and decrease loneliness. Aging \& Mental Health. 2007; 11(6):716-21. doi: $10.1080 / 13607860701366228$

[20] Hemati Alamdarlo G, Dehshiri GH, Shojaee S, Hakimirad E. Health and loneliness status of the elderly living in nursing homes versus those living with their families (Persian)]. Iranian Journal of Ageing. 2008; 3(2):557-68.

[21] Kwang YS, Yung GM. A study on the health statue and the needs of health- related services of Female elderly in an; urban - rural combined city Korea. Community Health Nursing Academic Society. 2003; 17(1):47-57.

[22] Sun WP. State and policy issues of home aged welfare service. Journal of the Korea Gerontological Society. 2001; 4:29-33.

[23] Asakawa K, Feeny D, Senthilselvan A, Johnson JA, Rolfson D. Do the determinants of health differ between people living in the community and in institutions? Social Science \& Medicine. 2009; 69(3):345-53. doi: 10.1016/j.socscimed.2009.05.007

[24] Moeini B, Shafii F, Hidarnia A, Babaii GR, Birashk B, Allahverdipour $\mathrm{H}$. Perceived stress, self-efficacy and its relations to psychological well-being status in Iranian male high school students. Social Behavior \& Personality. 2008; 36(2):257-66. doi: 10.2224/ sbp.2008.36.2.257

[25] Ghasemi H, Harirchi M, Masnavi A, Rahgozar M, Akbarian M. Comparing quality of life between seniors living in families and institutionalized in nursing homes (Persian)]. Social Welfare Quarterly. 2011; 10(39):177-200.

[26] De Leo D, Diekstra RFW, Lonnqvist J, Lonnqvist J, Cleiren MHPD, Frisoni GB, et al. LEIPAD, an Internationally applicable instrument to assess quality of life in the elderly. Behavioral Medicine. 1998; 24(1):17-27. doi: 10.1080/08964289809596377

[27] Keefe FJ, Ahles TA, Porter LS, Sutton LM, McBride CM, Pope MS, et al. The self-efficacy of family caregivers for helping cancer patients manage pain at end-of-life. Pain. 2003; 103(1):157-62. doi: 10.1016/s0304-3959(02)00448-7

[28] Gilliam CM, Steffen AM. The relationship between caregiving self-efficacy and depressive symptoms in dementia family caregivers. Aging \& Mental Health. 2006; 10(2):79-86. doi: 10.1080/13607860500310658

[29] Holland JM, Thompson LW, Tzuang M, Gallagher-Thompson D. Psychosocial factors among Chinese American women dementia caregivers and their association with salivary cortisol: results of an exploratory study. Ageing International. 2010; 35(2):109-27. doi: $10.1007 /$ s12126-010-9057-0

[30] Bastani F, Ghasemi E, Negarandeh R, Haghani H. General selfefficacy among family's female caregiver of elderly with Alzheimer's disease (Persian)]. Hayat. 2012; 18(2):27-37.

[31] Mohammadi Shahbolaghi F. [Self-efficacy and caregiver strain in Alzheimer's caregivers in the city of Tehran (Persian)]. Iranian Journal of Ageing. 2006; 1(1):26-33.

[32] Middleton J, Tran Y, Craig A. Relationship between quality of life and self-efficacy in persons with spinal cord injuries. Archives of Physical Medicine and Rehabilitation. 2007; 88(12):1643-8. doi: 10.1016/j.apmr.2007.09.001
[33] Rafii F, Naseh L, Yadegari M. [Relationship between self-efficacy and quality of life in ostomates (Persian)]. Iran Journal of Nursing. 2012; 25(76):64-76.

[34] Brink E, Alsén P, Herlitz J, Kjellgren K, Cliffordson C. General self-efficacy and health-related quality of life after myocardial infarction. Psychology, Health \& Medicine. 2012; 17(3):346-55. doi: 10.1080/13548506.2011.608807

[35] Wu AMS, Tang CSK, Kwok TCY. Self-efficacy, health locus of control, and psychological distress in elderly Chinese women with chronic illnesses. Aging \& Mental Health. 2004; 8(1):21-8. doi: $10.1080 / 13607860310001613293$

[36] Su-Hui Chen. The relationships among nutrition self-efficacy, health locus of control in elderly [PhD thesis]. Austin: University of Texas at Austin. 2007.

[37] Rambod M, Peyravi H, Sareban MT, Rafii F, Hoseini F. [Selfefficacy in hemodialysis patients and its related factors (Persian)]. Advances in Nursing \& Midwifery. 2008; 18(62):29-34.

[38] Jafarzade Fakhari M, Behnam Voshani HR, Vahedian Shahroodi M. [The quality of life of the elderly in Sabzevar, Iran (Persian)]. Journal of Sabzevar University of Medical Sciences. 2010; 17(3):213-7.

[39] Ahmadi F, Alizadeh S, Faqihzadeh S. [Quality of life in Zahedan elderly population (Persian)]. Hayat. 2004; 10(22):61-67.

[40] Joqataei MT, Nejati V. [Assessment of Health Status of Elderly People in the City of Kashan (Persian)]. Iranian Journal of Ageing. 2006; 1(1):3-10

[41] Canbaz S, Tevpik Senter A, Dabak S, Peksen Y. The prevalence of chronic diseases and quality of life in elderly people in Samsun. Turkish Journal of Medical Sciences. 2003; 33:335-40.

[42] Strauss E von, Agüero-Torres H, Kåreholt I, Winblad B, Fratiglioni L. Women are more disabled in basic activities of daily living than men only in very advanced ages: A study on disability, morbidity, and mortality from the Kungsholmen Project. Journal of Clinical Epidemiology. 2003; 56(7):669-77. doi: 10.1016/s08954356(03)00089-1

[43] Lee TW, Ko IS, Lee KJ. Health promotion behaviors and quality of life among community-dwelling elderly in Korea: A crosssectional survey. International Journal of Nursing Studies. 2006; 43(3):293-300. doi: 10.1016/j.jpurstu.2005.06.009

[44] Lee H, Cho SH, Kim JH, Kim YK, Choo HI. Influence of self efficacy, social support and sense of community on health-related quality of life for middle-aged and elderly residents living in a rural community. Journal of Korean Academy of Nursing. 2014; 44(6):608. doi: 10.4040/jkan.2014.44.6.608

[45] McAuley E, Konopack JF, Motl RW, Morris KS, Doerksen SE, Rosengren KR. Physical activity and quality of life in older adults: Influence of health status and self-efficacy. Annals of Behavioral Medicine. 2006; 31(1):99-103. doi: 10.1207/s15324796abm3101_14 